\title{
MIMO Design of Active Noise Controllers for Car Interiors: Extending the Silenced Region at Higher Frequencies
}

\author{
Simon Berthilsson, Annea Barkefors and Mikael Sternad
}

\begin{abstract}
Active Noise Control can provide damping of low-frequency noise in car compartments by feedforward or feedback control. The complicated sound field in reverberant environments limits the spatial volume of efficient sound control. At higher frequencies, damping is in general possible only within a small volume around a control microphone when using a single loudspeaker to produce the control signal. We here investigate how the spatial volume of control, as well as the upper limiting frequency, can be increased by using MIMO feedforward control that uses all loudspeakers of a premium car HiFi system. The results indicate that performance is improved for narrowband as well as broadband noise, resulting in a considerable noise reduction within a $0.3 \times 0.3 \mathbf{~ m}$ area. Compared to a corresponding SISO design that uses one loudspeaker and is optimized for one measurement position, the measured noise damping is improved and the upper limit frequency for control over the whole area is more than doubled, from $200 \mathrm{~Hz}$ to $450 \mathrm{~Hz}$. The design requires high-precision identification of acoustic transfer functions from all involved loudspeakers as well as accurate modeling of noise paths.
\end{abstract}

\section{INTRODUCTION}

Active noise control produces noise reduction around a point in space by destructive interference. It complements passive damping, in that active control is most efficient at lower frequencies, while the efficiency of passive damping increases with frequency. Since acoustic paths are dynamic systems with frequency-dependent gains, the control system in general needs to be a dynamic system. The adjustment of noise control systems is complicated and has to a large extent relied on adaptive control methods [1], [2], [3], [4].

Noise is becoming an increasing problem in cars, partly because the push to lower fuel consumption by reducing weight tends to reduce the use of passive damping. The automotive industry has therefore shown increasing interest in active control. The problem has been studied since the late 1980s [5], [6], [7], but until recently only a few examples of active noise control systems existed in passenger vehicles.

These systems have been limited to the very lowest frequencies. To the best of our knowledge, significant damping over useful spatial volumes has not been reported for frequencies above $200 \mathrm{~Hz}$ in car interiors. Pushing this limiting frequency upward is of practical interest, since it would reduce the frequency range where both passive and active means remain problematic. A fundamental problem here is that car interiors are complicated reverberant acoustic environments. At high frequencies, above the Schroeder

This research has been supported by the Swedish Research Council under contract 90552701 and by Dirac Research AB, Uppsala, Sweden.

Signals and Systems, Uppsala University, Box 534, 75121 Uppsala, Sweden. $\{$ sibe, anba, ms\}esignal.uu.se frequency of the enclosure, sound fields can be modeled as random fields. ${ }^{1}$ In this domain, the radius of effective control becomes approximately $1 / 7$ wavelengths [9], which is e.g. $0.05 \mathrm{~m}$ at $1000 \mathrm{~Hz}$. At lower frequencies, the sound field is less irregular. The Schroeder frequency for car compartments is rather high, due to their small volume. For example, it is in the range $450-580 \mathrm{~Hz}$ in the car used for the experiments here. This opens up the possibility that the upper limiting frequency of active noise control might be increased significantly above $200 \mathrm{~Hz}$.

For this purpose, we have investigated systems with multiple microphones, but found them to be inefficient as a means to increase the area of significant noise reduction. A control system that requires a very large number of active microphones in the car compartment would also tend to be expensive and error-prone. We have instead focused on the use of multiple loudspeakers. There already exists a large number of loudspeakers in mid-range and high-end car audio systems, so their dual use also for noise control is of interest. Furthermore, we wish to reduce the need for continuous online adaptation of high-order transfer functions in an active noise control system. As a part of this study, we here focus on controller designs based on off-line identification of acoustic transfer functions, to investigate their feasibility.

The use of multiple loudspeakers to control sound fields has received significant interest over the past decade. Three classes of methods have been proposed: Wave Field Synthesis [10], [11], High Order Ambisonics [12] and multipoint Mean Square Error (MSE) designs, see e.g. [13]. Of these alternatives, Wave Field Synthesis is based on idealized assumptions on the acoustic environment that are never fulfilled in practice. High Order Ambisonics is a per-frequency design that cannot optimize under delay constraints, which is important for active noise control. Multipoint MSE design remains the most promising practical MIMO control strategy.

In the feedforward control case, the transfer functions from $L$ noise measurement signals (the target paths, or noise paths) and from $N$ control loudspeakers (the secondary paths) are measured at $M$ control points located within the volume to be silenced. A linear controller is designed with the aim that the transfer functions from the $L$ feedforward signals, via the controller and loudspeakers to the $M$ control points, should approximate the target paths, but with negative signs.

\footnotetext{
${ }^{1}$ The Schroeder frequency indicates the lowest frequency for which the sound field becomes very irregular and a statistical model of the sound field is applicable [8]. It is conventionally defined as $f_{S}=2000 \sqrt{T_{60} / V}$ where $T_{60}$ is the reverberation time until a damping of $60 \mathrm{~dB}$, in seconds, while $V$ is the volume of the enclosure in $\mathrm{m}^{3}$.
} 
We here utilize an MSE feedforward control strategy based on LQG or $\mathrm{H}_{2}$ optimal control, using a polynomial equations approach to the design of filters and regulators [14]. LQG methods are most commonly used in relation to feedback control, but can be used in various feedforward or combined feedback and feedforward control problems as well [16], [22]. Compared to state-space methods, such solutions offer increased structural insight and also have good numerical properties. Compared to time domain matrix-based MSE methods [15], we avoid the inversion of extremely large block-Toepliz matrices. The solution constitutes a special case of a more general design derived in [16]. It has recently been applied successfully to problems of equalizer design and sound field control, see [17] and [18].

After introducing the problem and the design technique in Section II, we investigate the suppression of an artificial noise source at the driver's seat of a car compartment, using all loudspeakers of the car stereo system as actuators in Section III. We investigate and compare designs applicable for narrowband as well as broadband noise.

\section{A. Notation}

The trace and transpose of a matrix $\mathbf{M}$ are denoted $\operatorname{tr} \mathbf{M}$ and $\mathbf{M}^{\prime}$, respectively. Causal FIR filters with real-valued coefficients $\left\{p_{n}\right\}$ are here represented by polynomials in the backward time shift operator $q^{-1}$

$$
P\left(q^{-1}\right)=p_{0}+p_{1} q^{-1}+\ldots+p_{n p} q^{-n p},
$$

where $v(t-1)=q^{-1} v(t)$ while $v(t+1)=q v(t)$ for discretetime signals $v(t)$. Polynomial matrices are represented by bold italics, $\boldsymbol{P}\left(q^{-1}\right)$, and rational matrices by calligraphic symbols such as $\mathcal{R}\left(q^{-1}\right)$. Arguments $\left(q^{-1}, q, z^{-1}\right.$ or $\left.z\right)$ are omitted where there is no risk of misunderstanding.

A rational matrix may be parameterized in terms of polynomial matrices as a matrix fraction description (MFD), either left MFD $\mathcal{R}=\boldsymbol{A}_{1}^{-1} \boldsymbol{B}_{1}$ or right MFD $\mathcal{R}=\boldsymbol{B}_{2} \boldsymbol{A}_{2}^{-1}$ [19]. Here, a square polynomial matrix $\boldsymbol{P}\left(q^{-1}\right)$ is denoted "stable" if all zeros of $\operatorname{det}\left[\boldsymbol{P}\left(z^{-1}\right)\right]$ are located in $|z|<1$. For a polynomial matrix $\boldsymbol{P}\left(q^{-1}\right)$, the corresponding conjugate matrix $\boldsymbol{P}_{*}(q)$ is defined as its conjugate transpose, with the forward shift operator $q$ substituted for $q^{-1}$ as argument in all polynomials.

\section{MIMO LQG FEEDFORWARD SOUND FIELD CONTROL}

A linear acoustic system is affected by $L$ measurable noise signals, represented by a column vector $r(t)$, that are to be used for feedforward control. The influence of these noise components is to be suppressed at $M$ control points (measurement positions), using a set of $N$ loudspeakers where, in general, $N<M$. The control points are assumed located in the volume to be silenced, spaced by distances less than the spatial Nyquist frequency of the highest frequency sound to be controlled.

\section{A. Model Parameterizations}

The acoustic channel from the $N$ control loudspeakers to the $M$ measurement positions, including transport delays, needs to be measured and modeled. It is represented by

$$
y(t)=\mathcal{H}\left(q^{-1}\right) u(t)=\boldsymbol{B}\left(q^{-1}\right) \boldsymbol{A}^{-1}\left(q^{-1}\right) u(t) .
$$

Here, the vector $u(t)$ represents loudspeaker input signals at discrete time $t$, while $y(t)$ is the sound sampled at the measurement positions. The $M \times N$ polynomial matrix $\boldsymbol{B}\left(q^{-1}\right)$ and the $N \times N$ stable polynomial matrix $\boldsymbol{A}\left(q^{-1}\right)$ are a right MFD representation. In the experiments and evaluations in Section III, we use FIR models, for which $\boldsymbol{A}\left(q^{-1}\right)=\mathbf{I}$.

The noise components at the measurement positions that are correlated with $r(t)$ will be described by noise paths modeled by a matrix of FIR filters,

$$
z(t)=q^{-d} \boldsymbol{D}\left(q^{-1}\right) r(t) .
$$

The elements of the $M \times L$ polynomial matrix $\boldsymbol{D}\left(q^{-1}\right)$ include transport delays. We have in $z(t)$ in (2) also added an optional design delay $d$ that is common for all positions. Spectral properties of $r(t)$ are furthermore represented by a stable vector-autoregressive model

$$
\boldsymbol{H}\left(q^{-1}\right) r(t)=e(t)
$$

where $e(t)$ is a zero mean discrete-time white noise sequence with covariance matrix $\mathbf{R}_{e}$.

\section{B. The Design Problem}

The LQG feedforward regulator is a linear filter with $L$ inputs and $N$ outputs

$$
u(t)=-\mathcal{R}\left(q^{-1}\right) r(t+d) .
$$

The corresponding control error vector is

$$
\varepsilon(t)=z(t)+y(t)=\left(q^{-d} \boldsymbol{D}-\boldsymbol{H} \mathcal{R}\right) r(t+d) .
$$

The control paths $\mathcal{H} \mathcal{R}$ are made to approximate the "target" $q^{-d} \boldsymbol{D}$ that represents the noise paths delayed by $d$ samples. Use of a higher delay results in a higher approximation fidelity, approaching that of the noncausal Wiener solution when $d \rightarrow \infty$. However, to compensate for this the regulator (4) needs to act on time-shifted (predicted) feedforward signals $r(t+d)$. For narrowband noise (3), predictions can be performed with high precision while this is not the case for broadband noise. We will therefore use $d \gg 0$ in designs for narrowband noise below, while $d=0$ will be used for broadband designs.

The aim is to minimize the scalar quadratic criterion

$$
\begin{aligned}
J & =E\left\{(\boldsymbol{V} \varepsilon(t))^{\prime} \boldsymbol{V} \varepsilon(t)+(\boldsymbol{W} u(t))^{\prime} \boldsymbol{W} u(t)\right\} \\
& =E\left\{\operatorname{tr}\left[\boldsymbol{V} \varepsilon(t)(\boldsymbol{V} \varepsilon(t))^{\prime}\right]+\operatorname{tr}\left[\boldsymbol{W} u(t)(\boldsymbol{W} u(t))^{\prime}\right]\right\},
\end{aligned}
$$

under constraints of stability and causality of $\mathcal{R}\left(q^{-1}\right){ }^{2}$ The expectation $E(\cdot)$ in (6) is taken with respect to the statistical properties of $r(t)$. The weighting $\boldsymbol{V}\left(q^{-1}\right)$ is an $M \times M$ polynomial matrix of full normal rank $M$. The

\footnotetext{
${ }^{2}$ The regulator is restricted to be "causal" in the sense that it does not utilize $r(t+k)$ for $k>d$.
} 
$N \times N$ polynomial matrix $\boldsymbol{W}\left(q^{-1}\right)$ can be used to focus the control energy into frequency ranges appropriate for the loudspeakers and into signal subspaces that are appropriate for the room and the sound system.

\section{The LQG Feedforward Controller}

The filter design problem formulated above becomes a special case of a MIMO LQG feedforward regulator design problem in input-output form discussed in [16]. A unique solution exists under the following assumption:

Assumption 1: There exists an $N \times N$ polynomial matrix $\boldsymbol{\beta}\left(q^{-1}\right)$ that satisfies the spectral factorization equation

$$
\beta_{*} \beta=B_{*} V_{*} V B+A_{*} W_{*} W A,
$$

and that is stable and has a nonsingular leading coefficient matrix $\boldsymbol{\beta}(0)$.

Assumption 1 implies that $\boldsymbol{\beta}^{-1}\left(q^{-1}\right)$ represents a stable and causal dynamic system. The right polynomial matrix spectral factor $\boldsymbol{\beta}\left(q^{-1}\right)$ obtained from (7) will furthermore be unique, up to an orthogonal left matrix factor. Assumption 1 is fulfilled under mild conditions, see [16]. For example, the use of a control signal penalty matrix $\boldsymbol{W}$, such that $\operatorname{det}\left[\boldsymbol{W}\left(z^{-1}\right)\right] \neq 0$ on the unit circle $|z|=1$, will guarantee it.

Under Assumption 1, the unique stable linear feedforward regulator (4) that minimizes the criterion (6) for a model (1), (2), (3) is given by

$$
u(t)=-\boldsymbol{\mathcal { R }}\left(q^{-1}\right) r(t+d)=-\boldsymbol{A} \boldsymbol{\beta}^{-1} \boldsymbol{Q} r(t+d),
$$

where the causal $N \times L$ polynomial matrix $\boldsymbol{Q}\left(q^{-1}\right)$ is, together with a noncausal $N \times L$ polynomial matrix $\boldsymbol{L}_{*}(q)$, obtained as the unique solution to the linear polynomial matrix equation (Diophantine equation)

$$
q^{-d} \boldsymbol{B}_{*} \boldsymbol{V}_{*} \boldsymbol{V D}=\boldsymbol{\beta}_{*} \boldsymbol{Q}+q \boldsymbol{L}_{*} \boldsymbol{H} .
$$

See Section 3.3 of [16] for a proof.

The design equations are the polynomial matrix spectral factorization (7) and the polynomial matrix Diophantine equation (9), both of which can be efficiently solved. See e.g. [20] for a survey of the spectral factorization problem. ${ }^{3}$

It can be noted that the matrix $\boldsymbol{A}^{-1}\left(q^{-1}\right)$ in the model (1) will be canceled directly by the left factor $\boldsymbol{A}\left(q^{-1}\right)$ of $\boldsymbol{\mathcal { R }}\left(q^{-1}\right)$. The factor $\boldsymbol{A}\left(q^{-1}\right) \boldsymbol{\beta}^{-1}\left(q^{-1}\right)$ in (8) is an $N \times N$ rational matrix with poles in the roots of $\operatorname{det}\left[\boldsymbol{\beta}\left(z^{-1}\right)\right]=0$. These poles are inside the unit circle, since $\boldsymbol{\beta}\left(q^{-1}\right)$ is stably invertible under Assumption 1. Likewise, this filter is causal, since the spectral factor has nonsingular leading coefficient matrix and is therefore causally invertible.

The factor $\boldsymbol{A}\left(q^{-1}\right) \boldsymbol{\beta}^{-1}\left(q^{-1}\right)$ in (8) remains unaffected by the noise path model (2). The polynomial matrix $\boldsymbol{Q}\left(q^{-1}\right)$ depends on this model since $\boldsymbol{D}\left(q^{-1}\right)$ and the noise spectral model $\boldsymbol{H}\left(q^{-1}\right)$ are present in the Diophantine equation (9).

The filter (8) represents an IIR filter with $L$ inputs and $N$ outputs, of typically very high order. When implementing

\footnotetext{
${ }^{3}$ The general problem outlined in [16] also requires the solution of polynomial matrix coprime factorizations. With the model parametrization used here, with FIR models in (2) and stable AR models in (3), no coprime factorizations are needed.
}

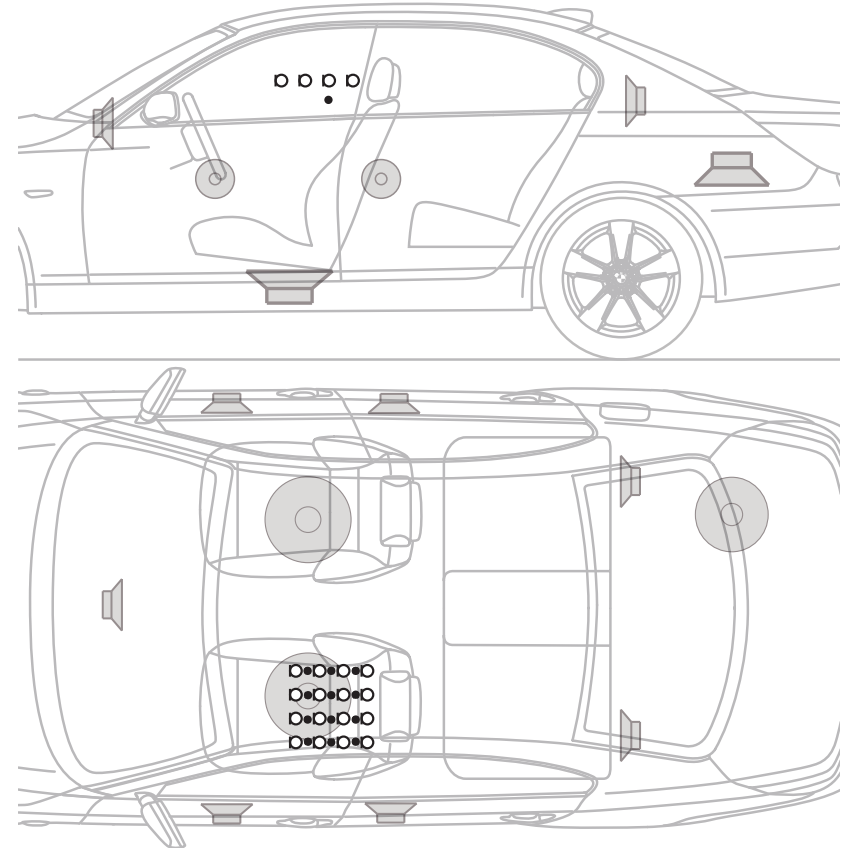

Fig. 1: Setup of the low- and mid-range loudspeakers and the microphone positions in the car. All the loudspeakers except the subwoofer in the trunk are the built-in loudspeakers. The ' $O$ ' symbols represent the microphone positions for design measurements, and the small ' $\bullet$ ' symbols represent the additional positions where verification measurements were performed.

it with finite precision arithmetrics, it can be advisable to approximate its transfer functions by high-order FIR filters. We have done so in the experiments described below.

\section{EXPERIMENTAL EVALUATION}

\section{A. Method and Assumptions}

All experiments presented in this paper were performed in a BMW 525i. A $4 \times 4$ grid of 16 equally spaced microphones, spanning $0.3 \times 0.3 \mathrm{~m}$ and placed at ear height in the driver's seat, as is shown in Fig. 1, were used to produce reference and verification measurements. In addition, 16 microphone positions off the original grid were used for verification measurements to see the effect outside the design points. Of these, 12 were placed between the measurement positions in the original measurement grid and four were placed on a row $0.1 \mathrm{~m}$ below said measurement grid. In Fig. 1 these are shown as small ' $\bullet$ ' symbols, whereas the original grid is indicated by ' $O$ ' symbols. Four omnidirectional microphones were used both for the design and for the evaluation.

The nine built-in low- and mid-range speakers were used as control speakers and a tenth subwoofer placed in the trunk acted as the noise source. The noise speaker was placed face down over a beam so as to couple to the body of the car, simulating noise originating from an engine. Fig. 2 shows the transfer functions of the noise speaker to the measurement grid. The noise speaker produces sound with significant energy up to $450 \mathrm{~Hz}$, with a distinct resonance due to the chassis around $43 \mathrm{~Hz}$. Since the measurement grid was placed in the driver's seat, the front left door speaker 


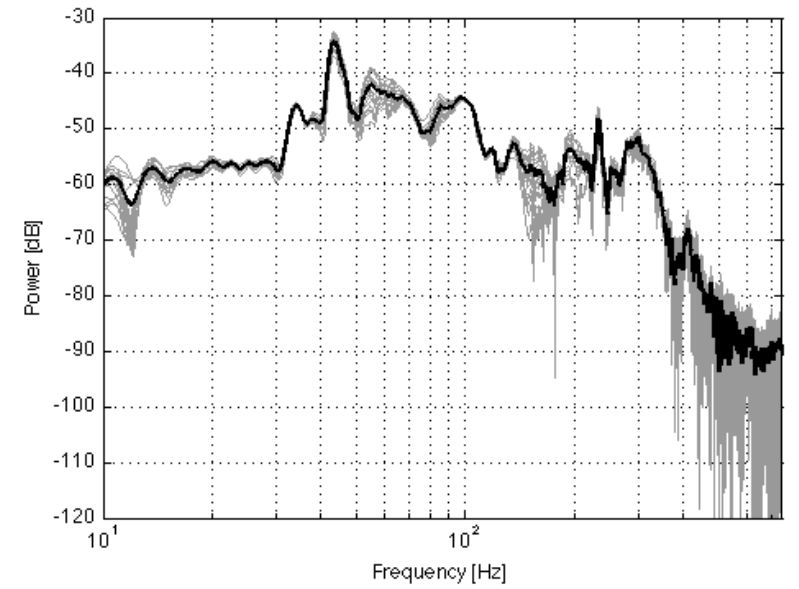

Fig. 2: Power Spectral Density plot of noise speaker at the 16 measurement points (gray). Also the RMS average over those points is shown (black).

with transfer function shown in Fig. 3 has the highest power gain of the mid-range speakers. At lower frequencies, below $80 \mathrm{~Hz}$, only the two subwoofers, that are located under the front seats, can be used for control. The spectral properties of the left subwoofer are shown in Fig. 4. Shown in Fig. 5 are the transfer functions from the left parcel shelf speaker. The parcel shelf speakers are of importance in that they are positioned close to the noise source and radiate sound with approximately the same angle of incidence as the nonbody-coupled sound waves from the noise speaker to the measurement grid.

One key precondition for reproducing the target well is that the phase of the target does not change irregularly within the volume to be silenced. For most frequencies, the phase changes smoothly over the measurement grid. A typical phase variation with position is illustrated (measured at the measurement points at a frequency of $300 \mathrm{~Hz}$ and interpolated between them to generate a surface) in Fig. 6 . Should the phase change irregularly over the volume to be silenced, additional degrees of freedom, i.e. loudspeakers may be needed in order to successfully solve the problem.

FIR models were adjusted to the impulse responses of the loudspeakers at the $0.3 \times 0.3 \mathrm{~m}$ microphone grid described above. Care was taken in order to preserve the properties of importance to the problem. In particular, the model orders (number of FIR coefficients) were selected to 938 samples at $1500 \mathrm{~Hz}$, allowing tails of the subwoofer impulse responses to be modeled accurately. Models were estimated using sine sweep signals as described in [21], repeated 9 times. Low frequency noise dominated the background noise with highest spectral density below $20 \mathrm{~Hz}$. The measurement noise contributes to the model errors by increased variance. The magnitude of this model error part can be estimated from differences in models produced at different time instants.

With these measurement-based models, (7) and (9) were solved to produce the optimal MIMO feedforward regulator $\boldsymbol{\mathcal { R }}\left(q^{-1}\right)$. The input signal to the noise loudspeaker acts as a

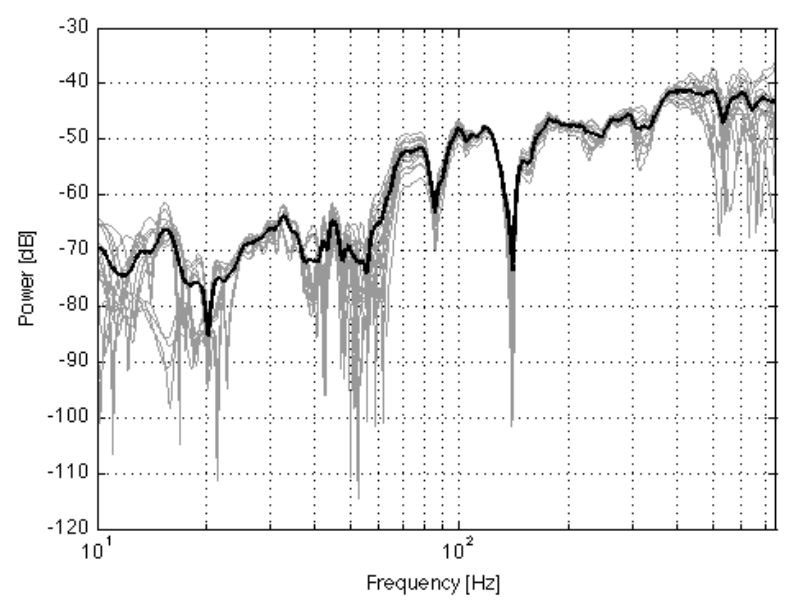

Fig. 3: Power Spectral Density plot of front left door speaker at the 16 measurement points (gray). Also the RMS average over those points is shown (black).

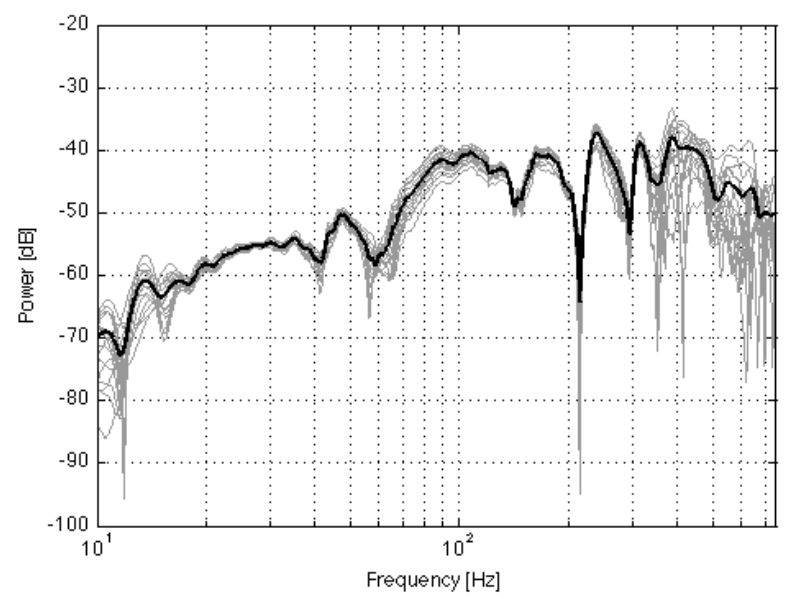

Fig. 4: Power Spectral Density plot of left subwoofer at the 16 measurement points (gray). Also the RMS average over those points is shown (black).

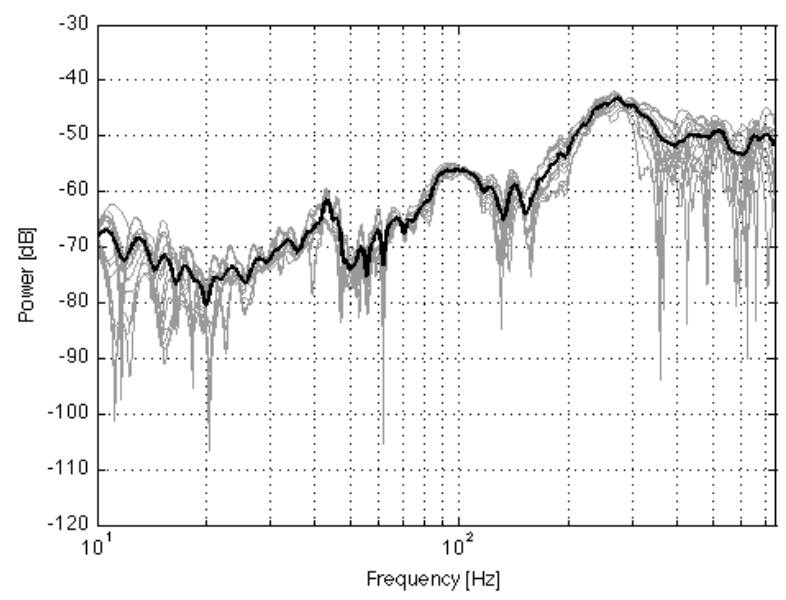

Fig. 5: Power Spectral Density plot of the left parcel shelf speaker at the 16 measurement points (gray). Also the RMS average over those points is shown (black). 


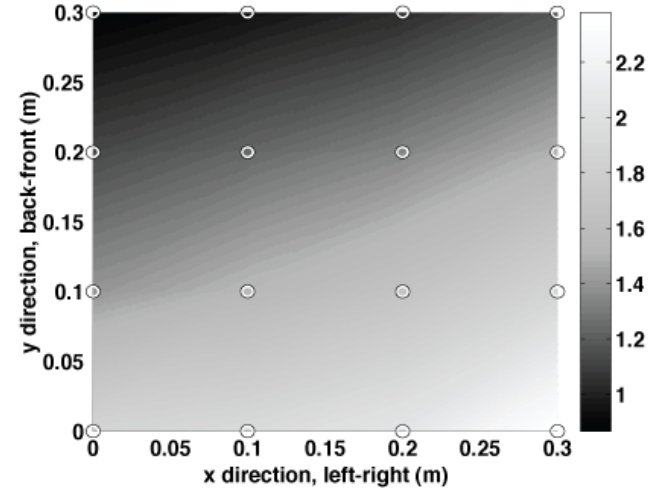

Fig. 6: The relative phase of sound originating from the noise speaker at $300 \mathrm{~Hz}$ over the measurement grid. On the $\mathrm{x}$ - and $y$-axes are the spatial dimensions of the grid while the shading represents the phase shift in radians, ranging from 0.9 radians in the front left corner of the grid to 2.4 radians in the rear right corner. Measurement positions are circled.

TABLE I: The frequency regions of low and mid-range loudspeakers and corresponding gains of the diagonal elements of the control signal weighting matrix $\boldsymbol{W}\left(q^{-1}\right)$

\begin{tabular}{|c||c||c||c|}
\hline Range & $\begin{array}{c}\text { Frequency region } \\
{[\mathrm{Hz}]}\end{array}$ & $\begin{array}{c}\text { Gain in region } \\
{[\mathrm{dB}]}\end{array}$ & $\begin{array}{c}\text { Gain outside region } \\
{[\mathrm{dB}]}\end{array}$ \\
\hline \hline Low- & $30-300$ & -30 & 0 \\
\hline Mid- & $80-500$ & -30 & 0 \\
\hline
\end{tabular}

measurable scalar feedforward signal $(L=1)$.

In order to keep the computational complexity down, all models and designs were measured and computed at a sampling frequency of $1500 \mathrm{~Hz}$. As mentioned in section II$\mathrm{B}$, different modeling delays $d$ were used for the broadband case and the narrowband case, resulting in two different designs. The broadband design used a modeling delay $d=0 \mathrm{~s}$, whereas in the narrowband design the modeling delay was $d=0.1 \mathrm{~s}$. In neither case the option of incorporating the relevant spectral properties of the source signal (see (3)) was used in the design process. This is equivalent to setting $\boldsymbol{H}$ in (3) and in (9) to unity.

The weighting matrix $\boldsymbol{V}\left(q^{-1}\right)$ in the criterion (6) was set to the unit matrix, since no microphone position was deemed more important than any other. The control signal weighting matrix $\boldsymbol{W}\left(q^{-1}\right)$ was selected diagonal and the filters in the diagonal elements were designed to have a low gain inside the operating region of the loudspeakers and a high gain outside it. The design is thus allowed to use the speakers only in their working frequency range. The weightings used in the MIMO designs for the different loudspeaker types throughout all the experiments are shown in Table I, together with the frequency regions of interest.

For comparison, a feedforward regulator was designed also for the simpler SISO case. One of the microphone positions was then targeted and controlled by the loudspeaker in the front left door. In this design, the spectral properties of the source signal were fully incorporated in the narrowband case, to maximize performance, giving rise to one design per tested frequency. For all the designs in the SISO case, the modeling delay $d$ was set to 0 and the scalar control penalty, $\boldsymbol{W}$ in the criterion (6), was set to $10^{-5}$.

Simulations were then performed to investigate how well the compensated control path matched the target (noise path). This was followed by validation measurements in the car. Measurements were also performed to see how well the designs attenuated a broadband noise with energy in the frequency region $30-300 \mathrm{~Hz}$, as well as single sinusoids with frequencies 100, 200, 300 and $350 \mathrm{~Hz}$.

\section{B. Results}

The results are divided into three categories. The first contains simulated results where the controller is applied to the system model used for design. The second describes the results of validation measurements in the car for the narrowband case, where a longer modeling delay can be afforded. The third shows the broadband validation results where low latency is of paramount importance and the modeling delay is reduced to zero.

The attained noise suppression in the validation measurements will depend on how well the system is able to reproduce the target function $q^{-d} \boldsymbol{D}\left(q^{-1}\right)$ of (2). The magnitude difference between the the error and the target function indicates the limit of achievable attenuation.

a) Simulations: Fig. 7a shows the simulated result of the narrowband design. An average damping in excess of $15 \mathrm{~dB}$ in the frequency regions $35-50 \mathrm{~Hz}$ and $90-450 \mathrm{~Hz}$ can be seen. Within $50-90 \mathrm{~Hz}$, the damping is lower, around $6-10 \mathrm{~dB}$. This is a frequency region where the target sound field shows increasing deviations between the measurement points, see Fig. 2. These are hard to reproduce exactly using only two subwoofers. In this frequency range, the mid-range speakers provide only a small contribution, see Table I.

Fig. $7 \mathrm{~b}$ shows the average result for the broadband design. The maximum achievable noise suppression is above $5 \mathrm{~dB}$ over the frequency range from $35 \mathrm{~Hz}$ up to $450 \mathrm{~Hz}$. Worth noting is that the most significant deterioration with respect to the narrowband case, arising from the use of a shorter modeling delay, is in the low frequency regions. This indicates that the approach may remain valid at the higher frequencies also in the more demanding broadband case.

b) Validation Measurements, Narrowband Results:

Fig. 7c shows the essentials of the narrowband validation results using feedforward control in the car. Test signal sweeps were run through the compensator and the loudspeakers and the result was measured at various microphone positions, see Fig. 1. Errors between the resulting transfer functions and their targets, represented by the elements of $z^{-d} \boldsymbol{D}\left(z^{-1}\right)$, are shown. Three zones may be discerned; the low frequency zone, below $30 \mathrm{~Hz}$, where no speakers are allowed to output energy according to the design. In this zone the model error is of the same magnitude as the target curve. The middle frequency zone, between $30 \mathrm{~Hz}$ and $80 \mathrm{~Hz}$ is defined by the two subwoofer speakers (see Table I); they manage to suppress the error fairly well. As in the corresponding simulations, the maximum noise suppression 
is reduced with increasing frequency since the speakers have difficulties controlling the increasingly complex sound field. From $80 \mathrm{~Hz}$ and upward, the mid-range speakers help the situation, defining the third zone with good attenuation properties. With increasing frequency comes increasing complexity and the error grows correspondingly. The locally poor performance around $150 \mathrm{~Hz}$ is in large explained by the noise transfer functions, showing significant spread in magnitude over the measurement points, see Fig. 2. This requires precise control, and small errors. However, in the frequency range $150-180 \mathrm{~Hz}$, the noise transfer functions to some measurement points also have deep notches. The relative estimation error due to the noise then becomes large at those notches. These model errors affect the design and contribute to the significantly worse validation results as compared to simulation results in this region. Above $180 \mathrm{~Hz}$ up to $450 \mathrm{~Hz}$, the achievable narrowband damping is again good, above $15 \mathrm{~dB}$ on average over the measured positions.

The design has also been validated by attenuating sinusoidal signals of 100, 200, 300 and $350 \mathrm{~Hz}$. The results from these experiments are shown in Table II (MIMO case) and in Table III (SISO case). For evaluation purposes, we define the relative difference in attenuation over the grid as

$$
\alpha=\frac{\max (\text { attenuation, } \mathrm{dB})-\min (\text { attenuation, } \mathrm{dB})}{\max (\text { attenuation, } \mathrm{dB})} .
$$

The relative difference in attenuation does not differ much between the MIMO and SISO experiments in the $100 \mathrm{~Hz}$ case. At $100 \mathrm{~Hz}$ we get $\alpha_{\text {MIMO }}=0.39$ and $\alpha_{\text {IISO }}=0.34$. There is on the other hand a large difference in the maximum attenuation between the two, $8.4 \mathrm{~dB}$ using the SISO design versus $23.9 \mathrm{~dB}$ with the MIMO approach. This is largely due to this particular SISO design being overly sensitive to model errors, causing the maximum attenuation to be reduced. ${ }^{4}$ Note however that this does not affect the wideness of the area of attenuation which is of primary interest here.

At $200 \mathrm{~Hz}, \alpha_{\text {мімо }}=0.23$ and $\alpha_{\text {sIso }}=0.87$, indicating an increase in evenness of the attenuation using the MIMO approach. Focusing on $300 \mathrm{~Hz}$, we note a rather striking difference: $\alpha_{\text {мімо }}=0.23$ and $\alpha_{\text {SISo }}=1.40$. The region of attenuation of the SISO design has here collapsed to the control point and its nearest neighbors. The MIMO design considers the whole grid and does therefore not amplify the noise in any point as the SISO design does. Last, at $350 \mathrm{~Hz}$ we get $\alpha_{\text {мIмо }}=0.309$ and $\alpha_{\text {SISO }}=1.551$.

In order to verify that the MIMO design produces reasonable results outside of the design points, verification measurements were performed in the positions indicated by ' $\bullet$ 's in Fig. 1. The results of this experiment is shown in Table IV and Table V. Finally, it was noted that no overtones were produced by the algorithms due to nonlinearities.

\footnotetext{
${ }^{4}$ The SISO regulator was designed based on the scalar counterparts to the matrices of Section II-B. It performs better than the MIMO design in its control point during simulations. However, if the control penalty, $\boldsymbol{W}$, is chosen low (as in the current SISO design) the result will be sensitive to model errors.
}

TABLE II: Attenuation of a sinusoidal signal in the $4 \times 4$ grid of control points when using MIMO LQG feedforward control

\begin{tabular}{|c||c||c||c|}
\hline \multicolumn{5}{|c|}{$100 \mathrm{~Hz}$} \\
\hline $15.5 \mathrm{~dB}$ & $16.0 \mathrm{~dB}$ & $15.8 \mathrm{~dB}$ & $14.7 \mathrm{~dB}$ \\
\hline $16.7 \mathrm{~dB}$ & $17.0 \mathrm{~dB}$ & $17.0 \mathrm{~dB}$ & $16.2 \mathrm{~dB}$ \\
\hline $21.5 \mathrm{~dB}$ & $21.2 \mathrm{~dB}$ & $21.6 \mathrm{~dB}$ & $21.0 \mathrm{~dB}$ \\
\hline $23.0 \mathrm{~dB}$ & $23.2 \mathrm{~dB}$ & $23.9 \mathrm{~dB}$ & $23.8 \mathrm{~dB}$ \\
\hline \hline \multicolumn{5}{|c|}{$200 \mathrm{~Hz}$} \\
\hline $16.4 \mathrm{~dB}$ & $16.1 \mathrm{~dB}$ & $15.7 \mathrm{~dB}$ & $14.6 \mathrm{~dB}$ \\
\hline $18.7 \mathrm{~dB}$ & $18.9 \mathrm{~dB}$ & $18.7 \mathrm{~dB}$ & $17.9 \mathrm{~dB}$ \\
\hline $16.4 \mathrm{~dB}$ & $16.9 \mathrm{~dB}$ & $17.2 \mathrm{~dB}$ & $17.3 \mathrm{~dB}$ \\
\hline $16.8 \mathrm{~dB}$ & $16.8 \mathrm{~dB}$ & $17.4 \mathrm{~dB}$ & $17.9 \mathrm{~dB}$ \\
\hline \hline \multicolumn{5}{|c|}{$300 \mathrm{~Hz}$} \\
\hline $23.2 \mathrm{~dB}$ & $23.0 \mathrm{~dB}$ & $22.7 \mathrm{~dB}$ & $22.0 \mathrm{~dB}$ \\
\hline $23.1 \mathrm{~dB}$ & $22.8 \mathrm{~dB}$ & $23.0 \mathrm{~dB}$ & $23.4 \mathrm{~dB}$ \\
\hline $22.8 \mathrm{~dB}$ & $22.7 \mathrm{~dB}$ & $22.9 \mathrm{~dB}$ & $23.5 \mathrm{~dB}$ \\
\hline $18.0 \mathrm{~dB}$ & $18.0 \mathrm{~dB}$ & $18.2 \mathrm{~dB}$ & $18.5 \mathrm{~dB}$ \\
\hline \hline \multicolumn{5}{|c|}{$350 \mathrm{~Hz}$} \\
\hline $15.7 \mathrm{~dB}$ & $14.5 \mathrm{~dB}$ & $13.0 \mathrm{~dB}$ & $15.0 \mathrm{~dB}$ \\
\hline $17.6 \mathrm{~dB}$ & $15.7 \mathrm{~dB}$ & $15.6 \mathrm{~dB}$ & $18.8 \mathrm{~dB}$ \\
\hline $16.1 \mathrm{~dB}$ & $15.1 \mathrm{~dB}$ & $13.8 \mathrm{~dB}$ & $16.8 \mathrm{~dB}$ \\
\hline $13.3 \mathrm{~dB}$ & $14.4 \mathrm{~dB}$ & $14.6 \mathrm{~dB}$ & $15.7 \mathrm{~dB}$ \\
\hline \multicolumn{4}{|c|}{}
\end{tabular}

TABLE III: Attenuation of a sinusoidal signal with SISO LQG designed for position $(4,2)$ (bold) in the $4 \times 4$ grid

\begin{tabular}{|c||c||c||c|}
\hline \multicolumn{5}{|c|}{$100 \mathrm{~Hz}$} \\
\hline $5.8 \mathrm{~dB}$ & $6.2 \mathrm{~dB}$ & $6.5 \mathrm{~dB}$ & $6.5 \mathrm{~dB}$ \\
\hline $6.7 \mathrm{~dB}$ & $7.0 \mathrm{~dB}$ & $7.1 \mathrm{~dB}$ & $6.8 \mathrm{~dB}$ \\
\hline $7.9 \mathrm{~dB}$ & $8.0 \mathrm{~dB}$ & $7.8 \mathrm{~dB}$ & $6.9 \mathrm{~dB}$ \\
\hline $8.8 \mathrm{~dB}$ & $\mathbf{8 . 4} \mathrm{dB}$ & $7.5 \mathrm{~dB}$ & $5.9 \mathrm{~dB}$ \\
\hline \hline \multicolumn{4}{|c|}{$200 \mathrm{~Hz}$} \\
\hline $1.3 \mathrm{~dB}$ & $3.3 \mathrm{~dB}$ & $6.0 \mathrm{~dB}$ & $9.8 \mathrm{~dB}$ \\
\hline $5.8 \mathrm{~dB}$ & $7.6 \mathrm{~dB}$ & $8.3 \mathrm{~dB}$ & $7.2 \mathrm{~dB}$ \\
\hline $6.3 \mathrm{~dB}$ & $6.8 \mathrm{~dB}$ & $6.4 \mathrm{~dB}$ & $4.9 \mathrm{~dB}$ \\
\hline $6.2 \mathrm{~dB}$ & $\mathbf{6 . 1 ~ d B}$ & $5.6 \mathrm{~dB}$ & $3.7 \mathrm{~dB}$ \\
\hline \hline \multicolumn{5}{|c|}{$300 \mathrm{~Hz}$} \\
\hline$-2.4 \mathrm{~dB}$ & $-1.9 \mathrm{~dB}$ & $-1.2 \mathrm{~dB}$ & $-0.1 \mathrm{~dB}$ \\
\hline$-1.4 \mathrm{~dB}$ & $-0.5 \mathrm{~dB}$ & $0.7 \mathrm{~dB}$ & $2.4 \mathrm{~dB}$ \\
\hline $0.2 \mathrm{~dB}$ & $1.8 \mathrm{~dB}$ & $3.5 \mathrm{~dB}$ & $5.1 \mathrm{~dB}$ \\
\hline $4.4 \mathrm{~dB}$ & $\mathbf{6 . 1} \mathrm{dB}$ & $5.8 \mathrm{~dB}$ & $2.2 \mathrm{~dB}$ \\
\hline \hline \multicolumn{5}{|c|}{$350 \mathrm{~Hz}$} \\
\hline$-1.4 \mathrm{~dB}$ & $-2.9 \mathrm{~dB}$ & $-3.7 \mathrm{~dB}$ & $-2.7 \mathrm{~dB}$ \\
\hline$-0.5 \mathrm{~dB}$ & $-1.0 \mathrm{~dB}$ & $-0.6 \mathrm{~dB}$ & $0.3 \mathrm{~dB}$ \\
\hline $1.3 \mathrm{~dB}$ & $2.0 \mathrm{~dB}$ & $2.6 \mathrm{~dB}$ & $2.8 \mathrm{~dB}$ \\
\hline $3.4 \mathrm{~dB}$ & $\mathbf{6 . 7} \mathrm{dB}$ & $6.1 \mathrm{~dB}$ & $4.3 \mathrm{~dB}$ \\
\hline
\end{tabular}

c) Validation Measurements, Broadband Results: The error power of the zero modeling delay MIMO design is plotted against the target curve in Fig. 7d. There is a reduction in performance of the broadband design compared to the narrowband design in Fig. 7c, as the modeling delay has been reduced. Note that the reduction of the control performance between the narrowband and the broadband cases is most prominent in the low frequency regions, mainly below $200 \mathrm{~Hz}$. This is most easily seen in the simulated results, Fig. 7a and Fig. 7b, but the effect is also readily discernible in the validation measurements, Fig. $7 \mathrm{c}$ and Fig. $7 \mathrm{~d}$.

The spectral content of the actual attenuation of a broadband noise with frequency content ranging from $30 \mathrm{~Hz}$ to $300 \mathrm{~Hz}$ is shown in Fig. 8. The result is similar to that produced by sine-sweep measurements in Fig. 7d. 


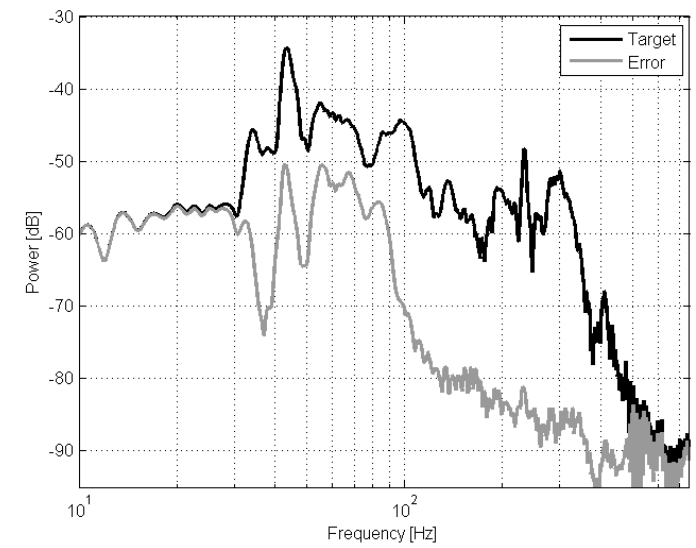

(a) Simulated result of the narrowband design. The target curve (black) is averaged over the 16 design points, as is the mean error curve (gray). The modeling delay $d$ is $0.1 \mathrm{~s}$.

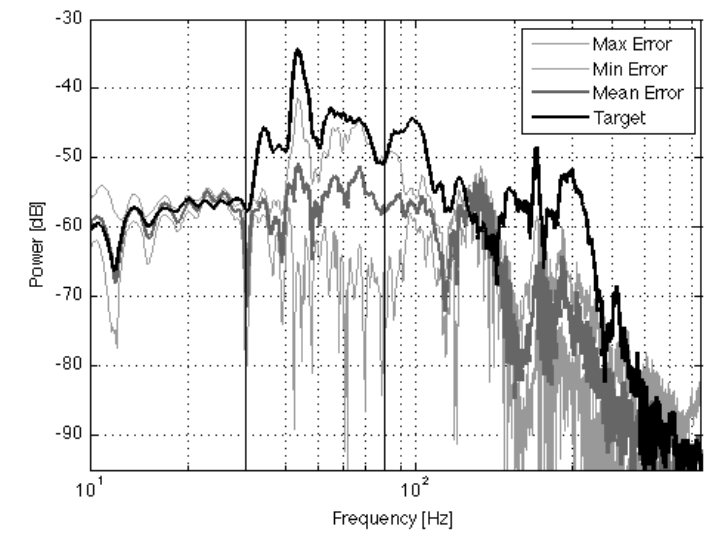

(c) Validation result of the narrowband design. The target curve is averaged over the entire measurement grid and plotted against the averaged error, the maximum error and the minimum error at al microphone positions. Three frequency zones discussed in the text are separated by vertical lines.

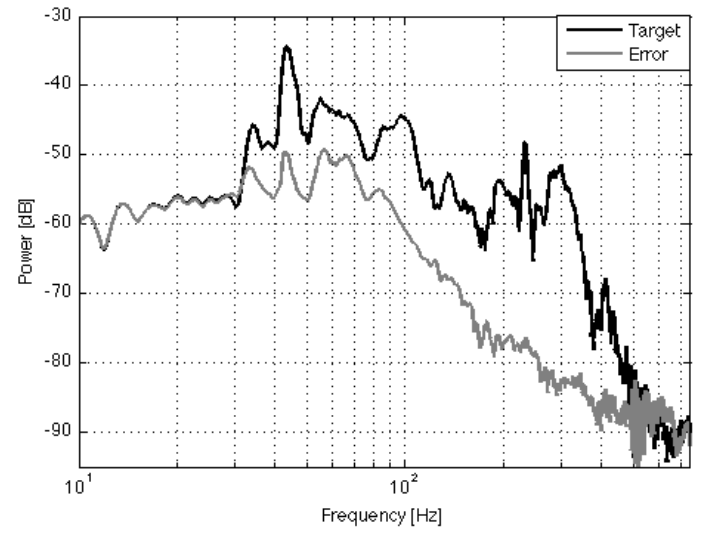

(b) Simulated result of the broadband design. The target curve (black) is averaged over the 16 design points, as is the mean error curve (gray). The modeling delay $d$ is $0 \mathrm{~s}$.

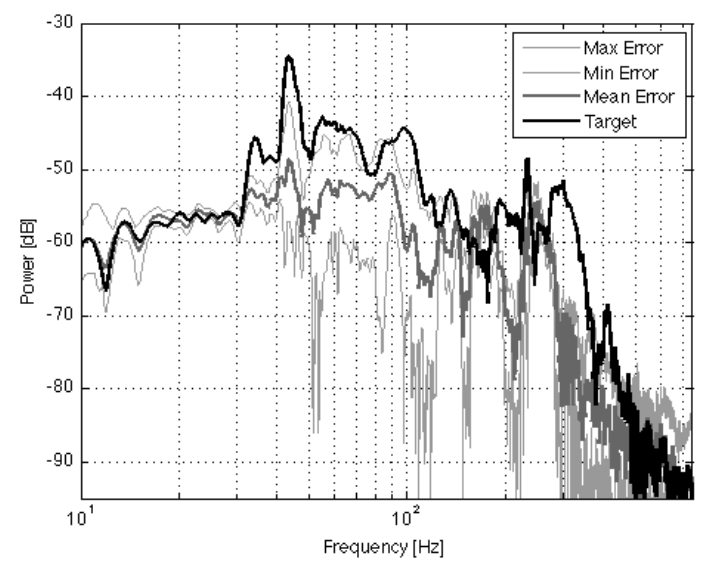

(d) Validation result of the broadband design. The target curve is averaged over the entire measurement grid and plotted against the averaged error, the maximum error and the minimum error at all microphone positions.

Fig. 7

TABLE IV: Attenuation of a $350 \mathrm{~Hz}$ signal at 12 positions between the control points when using MIMO LQG

\begin{tabular}{|c||c||c||c|}
\hline $15.8 \mathrm{~dB}$ & $15.2 \mathrm{~dB}$ & $14.2 \mathrm{~dB}$ & $16.8 \mathrm{~dB}$ \\
\hline $17.5 \mathrm{~dB}$ & $17.3 \mathrm{~dB}$ & $16.5 \mathrm{~dB}$ & $19.2 \mathrm{~dB}$ \\
\hline $14.9 \mathrm{~dB}$ & $15.7 \mathrm{~dB}$ & $14.2 \mathrm{~dB}$ & $15.7 \mathrm{~dB}$ \\
\hline
\end{tabular}

TABLE V: Attenuation of a $350 \mathrm{~Hz}$ signal with MIMO LQG $0.1 \mathrm{~m}$ below design plane

\begin{tabular}{|l||l||l||l|}
\hline $11.6 \mathrm{~dB}$ & $10.3 \mathrm{~dB}$ & $10.1 \mathrm{~dB}$ & $13.4 \mathrm{~dB}$ \\
\hline
\end{tabular}

\section{CONCLUSIONS}

This paper has shown that MIMO LQG control is a promising strategy for active noise control in car interiors. It has been verified that a good noise reduction performance can be obtained for $0.3 \times 0.3 \mathrm{~m}$ areas at the driver position over frequencies up to $450 \mathrm{~Hz}$, which is close to the Schroeder frequency of the car compartment. The
LQG feedforward design works well up to this frequency also without using a modeling delay. This is important in the suppression of wideband noise. The performance is improved below $200 \mathrm{~Hz}$ if a delay is allowed, which would be relevant in the narrowband noise case. The design is based on high-order models of transfer functions in FIR form, with realistically obtainable accuracy. It is worth pointing out that, as in many active noise control solutions, the overall sound level in the whole car compartment might be increased as opposed of decreased. What we have shown here is that we can reduce the noise level in a limited volume of space of our choice.

The present investigation was based on a single artificial noise source, in the form of an extra loudspeaker. An obvious important next step is to include multiple realistic feedforward signals such as undesired engine orders (overtones) in combination with road noise sensor signals, and study the modeling of their acoustic transfer functions. Another investigation of obvious interest is to simultaneously produce 


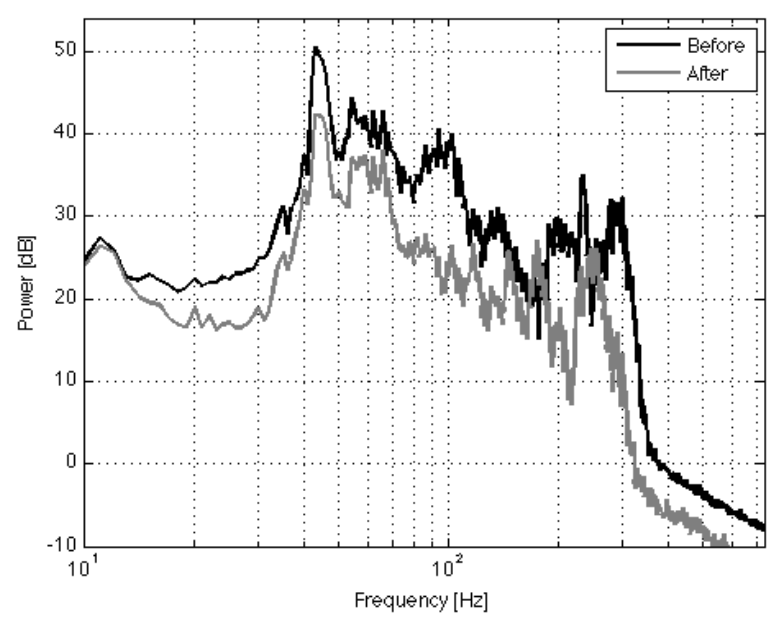

Fig. 8: Spectral content of the broadband attenuation using the MIMO controller for a noise ranging from 30 to $300 \mathrm{~Hz}$. The black curve shows the sound power without cancelation and the gray curve shows the sound power after cancelation.

silent zones at multiple seats. It is also of great interest to investigate the robustness of the method with respect to changes in the transfer functions. These changes may arise from e.g. passengers moving about in the car or windows being rolled down. A straightforward approach to produce a viable feedforward solution for different passenger constellations may be to simply generate a set of different filters (based on measurements with passengers or dummies). Switching between these different filters in the appropriate situations, e.g. based on the seat belt indicator pressure sensors, allows for a design tuned for each specific combination of occupants in the car. If the transfer functions are shown to vary in a way that makes the approach sketched above impractical, a feedback adaptation of the model will have to be considered.

Differences between simulations and validation measurements show the influence of model errors. In particular, the relative model errors become large in regions where the target transfer functions vary strongly between control points and where the control transfer functions have deep notches. It is of interest to generalize the LQG feedforward controller design to take model errors into account. The probabilistic robust (Bayesian) design proposed in [22] here offers a convenient alternative. This method has recently been used successfully in [18] and in [23] to introduce robustness at higher frequencies with respect to the variability of acoustic transfer functions in-between control points.

Acknowledgements. We thank Lars-Johan Brännmark for insightful comments on the results and for software tools used in this work.

\section{REFERENCES}

[1] S. J. Elliott and P. A. Nelson, "Active noise control," IEEE Signal Processing Magazine, vol. 10, pp. 12-35, Oct. 1993.

[2] P. A. Nelson and S. J. Elliott, Active Control of Sound. Academic Press, San Diego, 1992.
[3] S. M. Kuo and D. R. Morgan, Active Noise Control Systems Algorithms and DSP Implementations. Wiley, New York, 1996.

[4] S. M. Kuo and D. R. Morgan, "Active noise control: A tutorial review," Proceedings of the IEEE, vol. 87, no. 6, June 1999, pp. 943-973.

[5] S. J. Elliott and P. A. Nelson, "Multichannel active sound control using adaptive filtering," IEEE ICASSP 1988, pp. 2590-2593.

[6] T. J. Sutton, S. J. Elliott, A. M. McDonald and T. J. Saunders, "Active noise control of road noise inside vehicles," Noise Control Engineering Journal, vol. 42, pp. 137-147, July-Aug. 1994.

[7] H. Sano, T. Inoue, A. Takahashi, K. Terai, and Y. Nakamura, "Active control system for low-frequency road noise combined with an audio system," IEEE Trans. on Speech and Audio Processing, vol. 9, no. 7, Oct. 2001, pp. 755-763.

[8] M. R. Schroeder, "Statistical parameters of the frequency response curves of large rooms," Journal of the Audio Engineering Society, vol. 35, no. 5, pp. 299-306, May 1987.

[9] B. D. Radlovic, R. C. Williamson, and R. A. Kennedy, "Equalization in an acoustic reverberant environment: robustness results," IEEE Trans. on Speech and Audio Processing, vol. 8, no. 3, pp. 311-319, May 2000.

[10] S. Spors, R. Rabenstein and J. Ahrens, "The theory of wave field synthesis revisited", Presented at AES 124th Convention, Amsterdam, preprint 7358, May 2008.

[11] E. Corteel, "Equalization in an extended area using multichannel equalization and wave field synthesis," J. Audio Eng. Soc., vol. 54, no. 12, pp. 1140-1161, December 2006.

[12] M. A. Poletti, "Three-dimensional surround sound systems based on spherical harmonics," J. Audio Eng. Soc., vol. 53, no. 11, pp. 1104-1025, Nov. 2005.

[13] S. J. Elliott and P. A. Nelson, "Multiple-point equalization in a room using adaptive digital filters," J. Audio Eng. Soc., vol. 37, no. 11, pp. 899-907, November 1989.

[14] A. Ahlén and M. Sternad, "Wiener filter design using polynomial equations", IEEE Transactions on Signal Processing, vol. 39, pp. 2387-2399, 1991.

[15] Y. Huang, J. Benesty and J. Chen, Acoustic MIMO Signal Processing, Springer, New York, 2006.

[16] M. Sternad and A. Ahlén, LQ Controller Design and Selftuning Control. Chapter 3 in K. Hunt Ed, Polynomial Methods in Optimal Control and Filtering, Control Engineering Series, Peter Peregrinus, London, Jan. 1993, pp. 56-92.

[17] M. Johansson, L-J Brännmark, A. Bahne and M. Sternad, "Sound field control using a limited number of loudspeakers," AES 36th International Conference, Dearborn, Michigan, US, June 2-4 2009.

[18] L-J Brännmark, Robust Sound Field Control for Audio Reproduction: A Polynomial Approach to Discrete-Time Acoustic Modeling and Filter Design. Ph.D Thesis, Dept. of Engineering Sciences, Uppsala University, January 2011.

[19] T. Kailath, Linear Systems, Prentice-Hall, Englewood Cliffs, New Jersey, 1980.

[20] V. Kǔcera, "Factorization of rational spectral matrices: a survey of methods", IEE Control 91, Edinburgh, pp. 1074 1078, 1991.

[21] S. Müller and P. Massarani, "Transfer-function measurement with sweeps," J. Audio Eng. Soc., vol. 49, no. 6, pp. 443-471, June 2001

[22] K. Öhrn, A. Ahlén and M. Sternad, "A probabilistic approach to multivariable robust filtering and open-loop control," IEEE Trans. on Autom. Cont., vol. 40, March 1995, pp. 405-418.

[23] L-J Brännmark, "Robust audio precompensation with probabilistic modeling of transfer function variability," 2009 IEEE Workshop on Applications of Signal Processing to Audio and Acoustics (WASPAA 2009), Oct. 2009 New York City, NY. 